\title{
Study Of 50 Cases With Craniofacial Trauma Who Experienced Head Injuries
}

Ali Mesgarzadeh ${ }^{1}$, Mohammad Asghari ${ }^{2}$, Firooz Salehpoor ${ }^{2}$, Ailin Mahdkhah ${ }^{1}$, Armin Gosili ${ }^{1}$, Javad Aghazade $^{2}$, Mohammad Kazemzadeh ${ }^{2}$, Saba Asghari ${ }^{3}$ and Atta Mahdkhah ${ }^{2 *}$

1. Department of Oral and Maxillofacial Surgery, Tabriz University of Medical Sciences, Tabriz, Iran

2. Department of Neurosurgery, Tabriz University of Medical Sciences, Tabriz, Iran

3. Department of Ophthalmology, Tabriz University of Medical Sciences, Tabriz, Irane

\begin{abstract}
Background: It has been shown that cranial injuries associated with facial fractures may cause a great risk of mortality and neurological morbidity, which mainly occurs in young adults. Aims and objectives: Study of the features of facial injuries associated with head injuries, discussing the management options and detecting the outcomes following craniofacial trauma. Methods: This is a retrospective study carried out at Imam reza and Shohada Hospitals. Radiographs and hospital data of 50 patients with craniofacial trauma between January 2013 and December 2014, managed at the Oral and Maxillofacial surgery department were gathered and analyzed. Results: The greatest number of the patients had 20 to 50 years old $(68 \%)$ and most of them were male. (M/F ratio was $6.09: 1)$. The most prevalent causes of the trauma in this study were the motor vehicle accidents (44\%) and falling from height (36\%), respectively. The most common bone fracture among the patients was the zygomatic bone $\mathrm{fx}(38.2 \%)$. Among the symptoms which the patients presented, Loss of the consciousness (52\%) and headache (43\%) showed the highest prevalence. Compound depressed fractures, contusions and intracranial hematoma were the leading causes of the surgical intervention for intracranial lesions. A high number of patients who have died in this study had associated systemic injuries. Displaced facial bone fracture were the indications for operation in facial fractures. Conclusions: The majority of the patients with craniofacial trauma were the adult males and the leading cause of trauma were road traffic accidents. A high number of the patients had mild head injuries and required only a conservational therapy.
\end{abstract}

Keywords: head injury; craniofacial trauma; facial fracture

\section{Introduction}

It has been shown that cranial injuries and facial fractures have a great risk of mortality and morbidity, which occurs mainly in young adults. Oral Maxillofacial surgeons should be aware of these conditions and their management, because of a high incidence of closed head injuries and their potential for mortality and morbidity (1). Obviously, its so

\footnotetext{
Corresponding author:

Atta Mahdkhah

Department of Neurosurgery, Tabriz University of Medical Sciences, Tabriz, Iran

Email: mahdkhah@yahoo.com
}

Receive date: 2015-09-15 | Accept date: 2015-10-02 | Publish date: 2015-12-01

DOI: 10.7575/aiac.abcmed.16.04.01.04 
important for health care providers to perceive the patterns of facial injuries to efficiently treat and manage them. There is no need to say that, this tremendous epidemiologic data can be used for establishing a precise health program aimed towards prevention. In the present study, we have tried to analyze the clinical features, epidemiology and treatment options in craniofacial traumatic patients.

This study was designed to analyze the patterns and characteristics of cranio facial injury in head injury patients and gathering the information about possible outcomes and management options.

\section{Materials and methods}

This is a retrospective study performed at Imam reza and Shohada hospitals. A number of 50 sequential patients with craniofacial trauma managed at the department of the oral and maxillofacial surgery of Tabriz University of medical sciences between January 2013 and December 2014, selected and their radiographs and hospital data gathered and reviewed, following ethical committee approval. All clinical records reviewed and the data about age, sex, location and pattern of the injuries, concomitant cranial injury, treatment modalities and complications gathered and analyzed. According to the period of the LOC and amnesia and GCS, patients classified into 3 grades: mild, moderate and severe. (We have used Ommaya classification of head injuries in this study) (2). The trauma which involves craniofacial skeleton from the frontal bone to the mandible is described as maxillofacial trauma. Cranial fractures were categorized into 5 groups: occipital, temporal, parietal, Sphenoid and frontal. Fractures of the facial skeleton were classified into 3 groups: lower face (which refers to mandible), Midface (which refers to maxilla, nose, zygoma and orbits) and upper face (which refers to the frontal bone). Systemic injuries were categorized in this groups: abrasions and lacerations, hemothorax and pneumothorax, injuries to liver, kidney, bladder and bowels, loss of vision, fractures, long bone fractures, fracture, and spinal cord injury. CT scan was done for the patients who had a possibility of intracranial injury and/or facial bone fractures according to the sign and symptoms at the time of the admission. The definitive diagnosis of the facial fracture was underwent by attending radiologist. The Neurosurgery department and the oral and maxillofacial surgery departments were responsible for the management of the brain trauma and complex facial fractures, respectively.

\section{Results}

In the present study, there were a total number of 50 patients with cranial and/or maxillofacial injuries and most of them were men. $80 \%$ of the patients were male and $14 \%$ were female, in other words Male to female ratio was: 6.09:1. and a great number of them were between the ages of 20 and 50 (68\%). 5\% of the patients were under 10 and $18 \%$ of them had an age of 50 or higher. Motor vehicle accidents were the leading cause of the trauma (44\%) followed by fallings from height (36\%), violations and assaults (15\%), occupational injuries (5\%) and sports-related (1\%). The most frequent clinical symptom that the patients presented with was the loss of consciousness (52\%), followed by headache $(43 \%)$ and 3 patients have had an injury because of a train accident. Additionally, some of the patients were presented with vomiting (27\%), Epistaxis (23\%), and hematemesis (11\%). The leading site of the facial fractures was the zygomatic bone (38.2\%), followed by the mandibular fx. The maxillary bone was affected in $29.4 \%$ of the cases.

In this study, all the patients with moderate (4 cases- GCS 10-12) or severe (6 cases - GCS 69) head injuries had concomitant intracranial injuries and all the patients who sustained facial fractures had a mild head injury (i.e. GCS 
12-15). One of the patients had a spinal injury ( $f x$ of the L2 vertebra), Three of them had concomitant abdominal injuries (intestinal injury in 1 , hemoperitoneum in 1 , and splenic injury in 1). Five patients had orbital injuries and three of them sustained an orbital wall fracture. Five of the patients had an alcohol consumption history.

In the present study, most of the patients had a mild head injury (i.e. GCS 12-15) and were managed conservatively. Isolated undisplaced fractures of the mandible were managed conservatively with arch bar fixation. Here is the purposes that some of the patients had an operational intervention: compound depressed fracture of the frontal lobe (6 patients, who underwent wound debridement and internal fixation), decompression of left temporal contusion ( 4 patients) evacuation of an intracranial hematoma (extradural hematoma: 2 cases, chronic subdural hematoma: 4 cases) and suturing of scalp lacerations in 7 patients. Indications for an operational intervention for facial fractures were: displaced mandibular fractures (4 patients), zygomatic + maxilla fractures (4 cases), maxillary fractures (4 cases) and zygomatic fractures ( 3 patients). Al of the cases mentioned received internal fixation and open reduction; fixations were done by miniplates.

\section{Complications and mortality}

In the course of the present study, two of the patients died, one who had a concomitant splenic rupture and the other patient had a pulmonary injury which lead to hemothorax and aspiration. Additionally two of the patients suffered from wound infections and were managed conservatively.

\section{Discussion}

Majority of the patients with a head injuries accompanied by facial injuries in this study were among young adult males, as it is ranging from $3: 1$ to $11.1: 1$ in the literature $(3,4)$.
Maybe this is due to the occupational and outdoor harms which males are more suffering from because of involving in the activities and risk-taking jobs. In the most of the series motor vehicle accidents are the common cause of the craniofacial trauma $(6,7)$. It has been proven that the negligence and recklessness of the drivers, driving unresponsibly after the consumption of drugs or alcohol, ignoring the traffic laws and the poor quality of the vehicles, all have significant roles in occurrence of the road traffic accidents. As in this study, craniofacial trauma due to sports, violations, assaults and warfare is less common in the literature. But falling from height is one of the common causes of the injury in our series, incompatible with the most of other studies, and this can be assigned to the closeness of the hospitals to a coastal area where some people climb the coconut trees. In many areas violences and interpersonal assaults is being one of the major problems increasing the number of cranial injury patients and is attributed to the increasing use of alcohol and drugs (11). But in the present study there is not a significant number of these kind of cases. Maybe this is due the under report by the patients (12). Maxilla, orbit, and nasal bones were the most frequently fractured bones, among motorcyclists, followed by the floor of the orbit and nasal bones. But, in the present series, zygomatic bone was the most frequently fractured facial bone, followed by mandible and maxilla. High-velocity impacts can lead to the ruptures of intracranial vessels and fatal hemorrhages, As well as maxillofacial fractures (13-17). A Loss of the consciousness can be the symptom of a concussion head injury or intracranial injury; but, this is not much common with an isolated facial fractures. But in the present study, all the cases who had moderate to severe head injuries also had concomitant intracranial injuries showing the severity and complexity of the craniofacial trauma (18-20). 
In the present series, the majority of children had injuries mainly at home or playing places due to falling. Some other cases of children with facial injuries had road car accidents, escalator-related injuries, particularly in younger children, though we had not analyzed the causes of facial trauma in children, thoroughly.

In all the patients of craniofacial trauma, there should be a high index of suspicion of an associated cervical spine fracture as up to $10 \%$ of the patients can have concomitant spinal injuries. However the incidence of a concomitant spinal injury in the present series was low. There is no need to say that, the management and treatment of facial fractures should be delayed until more life-threatening problems have been managed, such as the hemodynamic stabilization, installation of a sufficient airway and treatment of other more serious injuries of the head, chest and skeleton.

It has been shown that the alcohol intoxication has a major role in worsening the consciousness of the patients with head injuries and a computed tomography is indicated when there is a history of alcohol consumption. An isolated mandibular fracture is the most common pattern of the facial fractures $(12.9 \%$ to as high as $72.9 \%$ ), followed by midface fractures (25.9\% to $29.5 \%$ ).

In literature, the incidence of post-surgical complications ranges from 5\%-5.7\%, including asymmetry, infection and malocclusion. In the present study there was a low incidence of the complications.

We have found out that most of the patients have mild head injuries and could be managed conservatively. Indications for surgical intervention include compound traumatic intracranial hematomas, depressed skull fractures, contusion and suturing of scalp lacerations.
In the present series, approaches to the facial fractures were according to the guidelines described in the literature, including repositioning of the displaced fracture segments into anatomic position, with a focus on the lattice supports in relation to each other and to the cranial base. Open reduction and internal fixation with miniplates was done in the cases of displaced fractures. Undisplaced fractures were managed conservatively and provided acceptable functional and aesthetic results. The rigid stabilization of the vertical and horizontal facial fracture. In order to help, support and withstand the forces of mastication, the rigid stabilization of the vertical and horizontal facial fracture was done. Mortality rate in our series was $2 \%$, and the major cause of this mortality was concomitant systemic injuries and pulmonary infection (21).

\section{Conclusions}

Although we have tremendous improvements in the management of the facial fractures, it remains a challenge for oral and maxillofacial surgeons, making it inevitable to use both skill and proficiency. In summary, majority of the head injuries occur among young male adults at motor vehicle accidents, but fortunately most of them have only a mild head injury. It's so important that we consider the priority of the management of the more life-threatening problems. For displaced facial bone fractures, open reduction and internal fixation with miniplates is recommended. We also suggest to design a well-organized prospective study to understand the patterns of facial injuries among the patients with head injuries. This clinical and epidemiological bank of data could pave the ground for health care providers to precisely plan the management options and goals in this patients and establishment of preventional health programs to reduce the impact of these injuries in the community. 


\section{References}

1. Conforti PJ, Haug RH, Likavec M. Management of closed head injury in patients with maxillofacial trauma. J Oral Maxillofac Surg. 1993;51:298-300.

2. Ommaya AK, Rockoff SD, Baldwin N. Experimental concussion. J Neurosurg. 1964;21:249-65.

3. Plaisier BR, Punjabi AP, Super Dm, Haug RH. The relationship between facial fractures and death from neurologic injury. J Oral Maxillofac Surg. 2000;58:708-12.

4. Zargar M, Khaji A, Karbakhsh M, Zarei MR. Epidemiology study of facial injuries during a 13 month of trauma registry in Tehran. Indian J Med Sci. 2004;58:109-14.

5. Bataineh AB. Etiology and incidence of maxillofacial fractures in the north of Jordan. Oral Surg Oral Med Oral Pathol Oral Rehabil Radiol Endod. 1998;86:31-5.

6. Ugboko VI, Odusanya SA, Fagade OO. Maxillofacial fractures in a semi-urban Nigerian teaching hospital: A review of 442 cases. Int J Oral Maxillofac Surg. 1998;27:286-9.

7. Marker P, Nielsen A, Bastian HL. Factures of the mandibular condyle, Part 1: Patterns of distribution of types and causes of fractures in 348 patients. Br J Oral Maxillofac Surg. 2000;38:417-21.

8. Haug RH, Prather J, Indresano AT. An Epidemiologic survey of facial fractures and concomitant injuries. J Oral Maxillofac Surg. 1990;48:926-32.

9. Ellis E, 3rd, Moos KF, El-Attar A. Ten years of mandibular fractures: An analysis of 2137 cases. Oral Surg. 1985;59:120.

10. Al Ahmed HE, Jaber MA, Abu Fanas SH, Karas M. The pattern of maxillofacial fractures in Sharjah, United Arab Emirates: A review of 230 case. Oral Surg Oral Med Oral Pathol Oral Radiol Endod. 2004;98:166-70.

11. Lynne HY, Kumar M, Charrles MM. Head and neck trauma in hospitalized pediatric patients. Otolaryngol Head Neck Surg. 2004;130:255-61.

12. Down KE, Boot DA, Gorman DF. Maxillofacial and associated injuries in severely traumatized patients: Implications of a regional survey. Int J Oral Maxillofac Surg. 1995;24:409-12.

13. Hung YC, Montazem A, Costello MA. The correlation between mandible fractures and loss of Consciousness. J Oral Maxillofac Surg. 2004;62:938-42.

14. Olson RA, Fonseca RJ, Zeitler DL. Fractures of the mandible: A review of 580 cases. J Oral Maxillofac Surg. 1982;40:23.

15. Ajagbe HÁ, Daramola JO, Oluwasanmi JO. Civilian-type facial injuries. A retrospective study of cases seen at the University College Hospital, Ibadan, Nigeria. Niger Med J. 1977;7:432-6.

16. Motamedi MH. An assessment of maxillofacial fractures: A 5-year study of 237 patients. J Oral Maxillofac Surg. 2003;61:61-4.

17. Güzel A, Karasalihoglu S, Küçükugurluoglu Y. Evaluation of the fall-related trauma cases applied to our pediatric emergency department. Ulus Travma Acil Cerrahi Derg. 2007;13:211-6.

18. Bulut M, Koksal O, Korkmaz A, Turan M, Ozguc H. Childhood falls: Characteristics, outcome, and comparison of the injury severity score and new injury severity score. Emerg Med J. 2006;23:540-5.

19. Montovani JC, de Campos LM, Gomes MA, de Moraes VR, Ferreira FD, Nogueira EA. Etiology and incidence facial fractures in children and adults. [Article in Portuguese] Rev Bras Otorrinolaringol (Engl Ed) 2006;72:235-41.

20. McGeehan J, Shields BJ, Wilkins JR, 3rd, Ferketich AK, Smith GA. Escalator-related injuries among children in the United States, 1990-2002. Pediatrics. 2006;118:e279-85.

21. Lewandowski B, Szymańska J. Agriculture-related severe craniofacial injuries in rural children and adolescents. Ann Agric Environ Med. 2008;15:59-62. 